\title{
COKRIGAGEM NA ESTIMATIVA ESPACIAL DA UMIDADE RELATIVA DO AR PARA O ESTADO DA BAHIA, BRASIL
}

\author{
Samira Luns Hatum de Almeida ${ }^{1}$ \\ Vinícius Agnolette Capelini \\ Jorge Tadeu Fim Rosas ${ }^{3}$ \\ Gabriel Dias de Oliveira ${ }^{4}$ \\ Julião Soares de Souza Lima ${ }^{5}$ \\ Samuel de Assis Silva ${ }^{6}$
}

Resumo: A estimativa da umidade relativa do ar para o Estado da Bahia é de grande importância para a atividade agrícola praticada na região, uma vez que essa variável influência em vários aspectos da cultura e também no bem-estar animal, já que a região possui uma forte atividade agropecuária. Com este trabalho se objetivou estimar a umidade relativa do ar no Estado da Bahia por meio da geoestatística, a partir de sua relação com a altitude, utilizando um método de interpolação multivariada, a cokrigagem. A cokrigagem permite obter estimativas mais precisas quando analisadas pares de variáveis. A utilização da altitude como variável auxiliar possibilitou bom desempenho para a interpolação por cokrigagem, sendo recomendada nesse tipo de estudo. A cokrigagem maximizou a variabilidade espacial da umidade relativa do ar no Estado da Bahia, reduzindo a continuidade do fenômeno principalmente nas regiões montanhosas do estado, na região próxima ao rio São Francisco e na divisa com os Estados de Pernambuco, Alagoas e Sergipe.

Palavras-chave: Geoestatística; Krigagem; Interpolador; Sistema de Informação Geográfica.

\footnotetext{
${ }^{1}$ Agronomia/Universidade Federal do Espírito Santo, Brasil. E-mail: samiraluns@hotmail.com.

2 Agronomia/Universidade Federal do Espírito Santo, Brasil. E-mail: vinicius91ac@hotmail.com.

3 Agronomia/Universidade Federal do Espírito Santo, Brasil. E-mail: jorgetadeufimrosas@hotmail.com.

${ }^{4}$ Agronomia/Universidade Federal do Espírito Santo, Brasil. E-mail: gabriel.dias.oliveira@gmail.com.

${ }^{5}$ Agronomia/Universidade Federal do Espírito Santo, Brasil. E-mail: limajss@yahoo.com.br.

${ }^{6}$ Agronomia/Universidade Federal do Espírito Santo, Brasil. E-mail: samuel.assilva@gmail.com.
} 